Draft VERSion SEPtember 26, 2018

Preprint typeset using $\mathrm{LAT}_{\mathrm{E}} \mathrm{X}$ style emulateapj v. 5/2/11

\title{
RESOLVED IMAGING OF THE HR 8799 DEBRIS DISK WITH HERSCHEL
}

\author{
Brenda C. Matthews ${ }^{1,2}$, Grant Kennedy ${ }^{3}$, Bruce Sibthorpe ${ }^{4}$, Mark Booth $^{5,2,1}$, Mark $^{2}$ Watt $^{3}$, \\ Hannah Broekhoven-Fiene ${ }^{2,1}$, Bruce Macintosh ${ }^{6,7}$, Christian Marois ${ }^{1,2}$ \\ Draft version September 26, 2018
}

\begin{abstract}
We present Herschel far-infrared and submillimeter maps of the debris disk associated with the HR 8799 planetary system. We resolve the outer disk emission at 70, 100, 160 and $250 \mu \mathrm{m}$ and detect the disk at 350 and $500 \mu \mathrm{m}$. A smooth model explains the observed disk emission well. We observe no obvious clumps or asymmetries associated with the trapping of planetesimals that is a potential consequence of planetary migration in the system. We estimate that the disk eccentricity must be $<0.1$. As in previous work by Su et al. (2009), we find a disk with three components: a warm inner component and two outer components, a planetesimal belt extending from 100 - 310 AU, with some flexibility $( \pm 10 \mathrm{AU})$ on the inner edge, and the external halo which extends to $\sim 2000$ AU. We measure the disk inclination to be $26 \pm 3^{\circ}$ from face-on at a position angle of $64^{\circ} \mathrm{E}$ of $\mathrm{N}$, establishing that the disk is coplanar with the star and planets. The SED of the disk is well fit by blackbody grains whose semi-major axes lie within the planetesimal belt, suggesting an absence of small grains. The wavelength at which the spectrum steepens from blackbody, $47 \pm 30 \mu \mathrm{m}$, however, is short compared to other A star debris disks, suggesting that there are atypically small grains likely populating the halo. The PACS longer wavelength data yield a lower disk color temperature than do MIPS data (24 and $70 \mu \mathrm{m}$ ), implying two distinct halo dust grain populations.
\end{abstract}

Subject headings: (stars:) circumstellar matter, planetary systems, individual (HR 8799)

\section{INTRODUCTION}

True Solar system analogues, systems of multiple planets with evidence for warm and cold dust disk components, have few representatives outside our own. A recently recognized exception is the HR 8799 (an A5V star at $39.9 \mathrm{pc}$ ) system which hosts a directly imaged multiplanet system and shows evidence for warm and cold dust disk components. Four planetary companions have been detected around HR 8799 at projected separations of 15, 24, 38 and 68 AU (Marois et al. 2008, 2010). Evidence for a circumstellar dust disk has been known for some time (Sadakane \& Nishida 1986), and Spitzer observations have revealed that the disk must contain multiple dust components ( $\mathrm{Su}$ et al. 2009; Reidemeister et al. 2009). Su et al. (2009) found evidence for three disk components: a warm central component, an outer "cold component" extending from 90 - 300 AU, and an external halo of small grains extending to an outer radius of 1000 AU. The relatively young age of this system (the most recent estimate gives an age of 20-50 Myr, Marois et al. 2010) makes it especially intriguing, since this is the cur-

\footnotetext{
${ }^{1}$ National Research Council of Canada Herzberg Astronomy \& Astrophsyics, 5071 W. Saanich Road, Victoria, BC, V9E 2E7, Canada

${ }_{2}^{2}$ Department of Physics \& Astronomy, University of Victoria, 3800 Finnerty Rd, Victoria, BC, V8P 5C2, Canada

${ }^{3}$ Institute of Astronomy, University of Cambridge, Madingley Road, Cambridge, United Kingdom, CB3 0HA

${ }^{4}$ SRON Netherlands Institute for Space Research, PO Box 800, NL-9700 AV Groningen, the Netherlands

${ }^{5}$ Instituto de Astrofísica, Pontificia Universidad Católica de Chile, Vicuña Mackenna 4860, 7820436 Macul, Santiago, Chile

${ }^{6}$ Lawrence Livermore National Labs, 7000 East Ave, Livermore, CA 94550, U.S.A.

7 Department of Physics \& Kavli Institute for Particle Astrophysics and Cosmology, Stanford University, Palo Alto, CA U.S.A.
}

Table 1

Observations Log

\begin{tabular}{cccc}
\hline \hline Obs. ID & Observing date & Mode & Duration \\
\hline $1342223848 / 9$ & 9/10 July 2011 & PacsPhoto 70/160 & $9018 \times 2$ \\
$1342223850 / 1$ & 10 July 2011 & PacsPhoto 100/160 & $7890 \times 2$ \\
$1342234924-28$ & 18 Dec 2011 & SpirePhoto & $169 \times 5$ \\
\hline
\end{tabular}

rently favored epoch for the formation of terrestrial planets (Chambers 2001; Ravmond et al. 2004; Melis et al. 2010).

We also have an ideal viewing angle toward this system, as the orbital plane of the planets and disk appear to lie very close to the plane of the sky (Marois et al. 2008; Lafrenière et al.|2009; Reidemeister et al. |2009; Su et al. 2009; Fabrycky \& Murrav-Clav 2010; Soummer et al. 2011), although the inclination of the star has been estimated to be in excess of $40^{\circ}$ by Wright et al. (2011). A face-on geometry makes it possible to observe azimuthal variations in the disk structure, as well as radial ones (e.g., $\epsilon$ Eri, Greaves et al. $(1998,2005)$ and Vega, Holland et al. (1998), though see Backman et al. (2009) and Hughes et al. (2012)). The presence of so many giant planets in a system makes it inevitable that the debris disk has been sculpted by gravitational perturbations. Wvatt et al. (1999) and Mouillet et al. (1997) show that the planets' secular perturbations could impose eccentricity or inclination on the disk (e.g., $\beta$ Pic, Lagrange et al. 2012), or migration of the planets could have trapped material into resonance (Wyatt 2003).

The combination of four massive, coeval, and spectroscopically characterizable planets (Konopacky et al. 2013; Bowler et al. 2010), together with the dust disk, makes this system a "Rosetta Stone" for planet formation. The three outer planets have significant grav- 
Table 2

Observations Summary

\begin{tabular}{ccccc}
\hline \hline $\begin{array}{c}\text { Wavelength } \\
{[\mu \mathrm{m}]}\end{array}$ & $\begin{array}{c}\text { FWHM } \\
{\left[{ }^{\prime \prime}\right]}\end{array}$ & $\begin{array}{c}\text { Integration time } \\
{[\text { hours }]}\end{array}$ & $\begin{array}{c}\text { instrumental noise } \\
{\left[\mathrm{mJy} \mathrm{beam}^{-1}\right]}\end{array}$ & $\begin{array}{c}1-\sigma \text { rms noise level } \\
{\left[\mathrm{mJy} \mathrm{beam}^{-1}\right]}\end{array}$ \\
\hline 70 & $5.5 \times 5.8$ & 5.1 & 0.4 & 0.338 \\
100 & $6.7 \times 6.9$ & 4.45 & 0.5 & 1.43 \\
160 & $10.7 \times 12.1$ & 9.55 & 0.6 & 8.90 \\
250 & $18.7 \times 17.5$ & 0.3 & 4.0 & 25.16 \\
350 & $25.6 \times 24.2$ & 0.3 & 3.4 & 29.01 \\
500 & $38.2 \times 34.6$ & 0.3 & 4.8 & 20.70 \\
\hline
\end{tabular}

itational interactions (Fabrvckv \& Murrav-Clav 2010; Reidemeister et al. 2009; Goździewski \& Migaszewski 2009, 2013); the age of the system requires it to be stable over $10^{8}$ year timescales. This in turn requires either a very finely tuned orbit for the nominal 5-7 $M_{J}$ planets, or low planet masses. Precision astrometry (Konopacky et al. 2009, 2010) being carried out at the Keck observatory can constrain these orbital scenarios even over partial orbits. However, the outermost planet, "b", with its long period, is the most weakly constrained. Future resolution of the inner edge of the planetesimal belt will provide significant constraints on outer-planet inclination given the coplanarity of the planets and disk discussed in this work. Resolving the inner edge will also provide constraint on the eccentricity of the disk to a simultaneous astrometric and dynamical fit. Finally, such high resolution images can provide mass information in the same way that the dust rings have been used around $\epsilon$ Eri (Quillen \& Thorndike 2002) and around Fomalhaut to infer an orbit and mass of Fomalhaut b, though it now appears to apply to a hypothetical Fomalhaut c (Chiang et al. 2009; Kalas et al. 2013).

We present higher resolution far-infrared data than was obtainable by Spitzer toward HR 8799 from the Herschel Space Observatory's PACS (Photodetector Array Camera and Spectrometer, Poglitsch et al. 2010) instrument at 70,100 and $160 \mu \mathrm{m}$. In addition, we present lower resolution submillimeter images from the SPIRE (Spectral and Photometric Imaging REceiver Griffin et al. 2010) camera at 250,350 and $500 \mu \mathrm{m}$. These data were taken during the Herschel Open Time Cycle 1 and reveal a well-resolved disk around HR 8799 in the far-infrared. We describe our observations in $\S 2$, describe the data reduction in $\S 3$ and present the data in $\S 4$. In $\S 5$, we present models of the resolved images. In $\S[$, we explore the implications of these data. We summarize our findings in $\S 7$

\section{OBSERVATIONS}

The record of the observing log is shown in Table 1. The PACS maps were made using the mini-scan mapping mode (Poglitsch et al. 2010) at a scan speed of $20^{\prime \prime} / \mathrm{s}$ across the target in 10 scan legs, separated by a scan line cross-step of $4^{\prime \prime}$. Each scan leg is $3^{\prime}$ long. At $70 \mu \mathrm{m}$, there are 64 repeats of each map, with the observations paired into two scanning angles: 70 and 110 degrees (with respect to the instrument reference frame). At $100 \mu \mathrm{m}, 56$ maps are required, with 28 at each of the two scanning angles specified above. The total integration time on the target is 5.1 hours with PACS 70/160 and 4.45 hours with PACS 100/160. The $160 \mu \mathrm{m}$ data were obtained at the same time as the $70 \mu \mathrm{m}$ and $100 \mu \mathrm{m}$ data. The com- bined $160 \mu \mathrm{m}$ map from both observations just reaches the $160 \mu \mathrm{m}$ confusion limit.

SPIRE observes all three submillimeter bands simultaneously to the confusion limit in 0.3 hour of time. Five maps were made in small-map mode with a dither set between each map of $12^{\prime \prime}$ (twice the nominal $250 \mu \mathrm{m}$ pixel scale). The concatenation of these observations provides a more uniform coverage of the disk emission than a single longer integration and allows us to use a finer pixel scale in the output maps.

The total mapped area of the PACS observations is $3.5^{\prime} \times 6.5^{\prime}$, but the area with high coverage, i.e., the "map center", corresponds to all regions that have at least $80 \%$ of the peak coverage, and high integration time is restricted to $1^{\prime} \times 2^{\prime}$. The details of the observations and the resulting rms noise estimates at each wavelength are summarized in Table 2. The high $\mathrm{S} / \mathrm{N}$ coverage area of the SPIRE maps is $4^{\prime} \times 4^{\prime}$.

Noise measurements are taken from within a $2^{\prime} \times 2^{\prime}$ area centred on the source. The noise is estimated by taking the RMS of a series of many summed apertures across the field, with each aperture having an area equal to that of a diffraction-limited Gaussian beam. Note that the high noise values at and longward of $160 \mu \mathrm{m}$ are a result of the high cirrus confusion in the region. The fact that the noise peaks at around $250-350 \mu \mathrm{m}$, i.e. the peak of the ISM dust emission, and then drops at $500 \mu \mathrm{m}$ is indicative that this is the primary source of noise at these wavelengths.

\section{DATA REDUCTION}

The data were reduced using version 7 of the Herschel interactive pipeline environment (HIPE Ott 2010) software, implementing versions 32 and 8.1 of the PACS and SPIRE calibration products respectively. The standard processing steps were followed, with an additional jackknife deglitching step implemented in the PACS data reduction, removing any artefacts with a significance greater than 5- $\sigma$ in the jack-knifed image. This step was performed as an additional deglitching precaution only.

The PACS time-line data were high-pass filtered to reduce the impact of $1 / f$ noise (see Section 3.1 for details), and converted to a map using the HIPE 'photProject' task. Maps were likewise made from the SPIRE data using the 'naiveMapper' task. No filtering was performed on the SPIRE data.

The maps were created with pixel scales a fraction of the beam size. The sizes used are $1^{\prime \prime}$ for all PACS maps, $3^{\prime \prime}$ for $250 \mu \mathrm{m}, 4^{\prime \prime}$ for $350 \mu \mathrm{m}$, and $5^{\prime \prime}$ for $500 \mu \mathrm{m}$. The SPIRE bands are highly oversampled due to the dithering used during observations, which allows us to produce maps with a relatively fine pixel scale. 


\subsection{Filtering effects}

During processing the PACS time-ordered data were high-pass filtered to mitigate low $1 / f$ frequency noise. Filtering was performed on each bolometer individually, passing all scales above a user-given input temporal scale. Initially blind filtering was performed and a map made from these filtered data. This map was used to identify all structure with a significance greater than or equal to $3-\sigma$, from which a filter mask is created. The original data were then filtered again, this time masking regions containing real structure, and the final map was projected. Masking of map sources is required to remove ringing style filter artefacts along the telescope scanning directions.

Even though sources in the map were masked during filtering, the expected extent and interest in the lowlevel emission from the diffuse outer disk meant that it was critical to quantify the impact of the filtering on the measured disk structure. For example, since the filtering is performed in the scan direction only, a small filter scale could truncate the disk along the scan direction, making a face-on disk appear to be inclined. Even though two scanning directions were used to perform these observations their separation was only $40^{\circ}$, making disk truncation a possibility. A large filter scale improves source fidelity, but it also passes equivalently larger angular scales to the map, resulting in an increased noise level.

We began by producing reference maps with a filter scale of $66^{\prime \prime}$ (16 frames), and generated additional maps using increasingly large filter scales. The integrated flux density on the source was then measured using a circular aperture with a radius of $30^{\prime \prime}$ in each map. The background was subtracted using an annulus centred on the source position, with inner and outer radii of $45^{\prime \prime}$ and $60^{\prime \prime}$ respectively. The measured flux density from each map was normalised to that measured in the reference map. The rate of change of normalised flux density with filter scale was initially high, and decreased as the filter scale became large relative to the size of the disk. Following this analysis we chose to use maps created with a filter scale of $402^{\prime \prime}$ (100 frames) where the rate of change was small for all bands.

While this larger filter scale results in an increased noise level, the increase in source flux is similar, meaning that there is little loss in signal-to-noise ratio. In addition, the improvement in source structure fidelity is significant, and necessary for accurate disk modelling.

\section{RESULTS}

Figure 1 shows the measured emission on the sky toward HR 8799. HR 8799 is detected at all wavelengths. A compact background source, also detected at 24 and $70 \mu \mathrm{m}$ by Su et al. (2009), is detected $\sim 15^{\prime \prime}$ northwest of the disk emission and is resolved from HR 8799 at all PACS wavelengths. The images from $160-500 \mu \mathrm{m}$ show asymmetric, large-scale emission in addition to the HR 8799 disk emission. Figure 2 shows an IRAS $100 \mu \mathrm{m}$ map of the sky around HR 8799. The significant large scale background emission underlying the HR 8799 position arises from a (most likely background given the proximity of HR 8799 at $39 \mathrm{pc}$ ) dust cloud. We have used our SPIRE data to make a temperature map of the large-scale emission by fitting SEDs to each pixel in the
SPIRE maps (convolved to common resolution), shown in Figure 3. The position of HR 8799 shows up as a small region of warmer dust amid the $\lesssim 20 \mathrm{~K}$ dust of the background cloud.

The emission from the HR 8799 disk is clearly resolved at all PACS wavelengths, but the impact of the underlying cirrus emission increasingly affects interpretation of the data at and longward of $160 \mu \mathrm{m}$.

\subsection{Photometry}

Emission from the HR 8799 system was measured using aperture photometry. These apertures are necessarily large to include as much disk emission as possible, so at 70, 100 and $160 \mu \mathrm{m}$ also include emission from the NE background source. We used apertures of 30, 30 and $26^{\prime \prime}$ for 70,100 and $160 \mu \mathrm{m}$, repectively. The aperture at $160 \mu \mathrm{m}$ was chosen to minimize the contribution from the large-scale background cloud, but is large enough to encompass the point-like background source. At this wavelength the high overall background level means that the flux density measured depends strongly on the aperture size, an issue we revisit below. Flux densities for the background source were estimated during the modelling process using PSF fitting and were subsequently subtracted from the aperture measurements.

An additional issue arises due to the high-pass filtering, which removes emission on large spatial scales. While we have shown that more disk emission is not gained by increasing the filter scale, it is likely that emission is still missing from the aperture photometry because the PACS beam has significant emission on large spatial scales. For example, the PACS documentation shows that at $100 \mu \mathrm{m}$ with a filter scale of $100^{\prime \prime}$, about $10 \%$ of the flux from a point source lies beyond $30^{\prime \prime}$. This emission cannot be detected with typical observation 8 because the extended emission is very faint and normalised out by filtering (i.e. the background sky level is set to zero). To account for this missing flux, we use the PACS aperture corrections of $0.87,0.88$, and 0.83 for 70,100 and $160 \mu \mathrm{m}$ (at 30,30, and $\left.26^{\prime \prime}\right)$.

The raw fluxes measured in each aperture are 472, 616, and $553 \mathrm{mJy}$, and the NE background source estimates are $4.7,11.8$, and $15.3 \mathrm{mJy}$ for 70,100 and $160 \mu \mathrm{m}$, respectively. Therefore, after subtracting the background sources and dividing by the aperture corrections, the fluxes are 537 and $687 \mathrm{mJy}$ at 70 and $100 \mu \mathrm{m}$, as shown in Table 3 and $648 \mathrm{mJy}$ at $160 \mu \mathrm{m}$. (We discuss the 160 $\mu \mathrm{m}$ entry to Table 3 in the next paragraph.) The PACS observations are fairly deep, so the uncertainties on these measurements are dominated by calibration $(2.64,2.75$, and $4.15 \%$ at 70,100 and $160 \mu \mathrm{m}$ respectively). We therefore adopt uncertainties of 15 and $20 \mathrm{mJy}$ at $70 \mu \mathrm{m}$ and $100 \mu \mathrm{m}$, respectively. Given these uncertainties the flux densities of the disk are detected at a $S / N$ of 35 at both wavelengths.

In modelling the observations below, we find that the flux density uncertainty is dominated by the background level at $160 \mu \mathrm{m}$ and longer wavelengths. The flux density increases with aperture size, suggesting that the 650 mJy flux density likely includes some of the extended

\footnotetext{
8 The extended emission was characterised by observing Mars, which was heavily saturated near the peak but allowed radial profiles of the outer parts of the beam profile to be derived.
} 


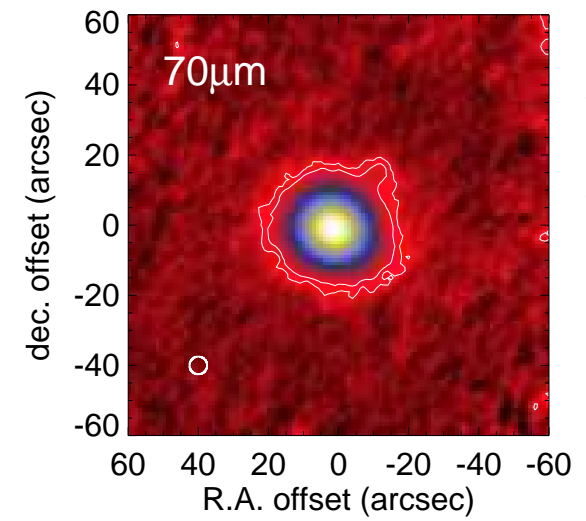

Surface Brightness (mJy/arcsec $\left.{ }^{2}\right)$

$\begin{array}{lllllll}-0.05 & 0.23 & 0.51 & 0.79 & 1.07 & 1.35 & 1.63\end{array}$

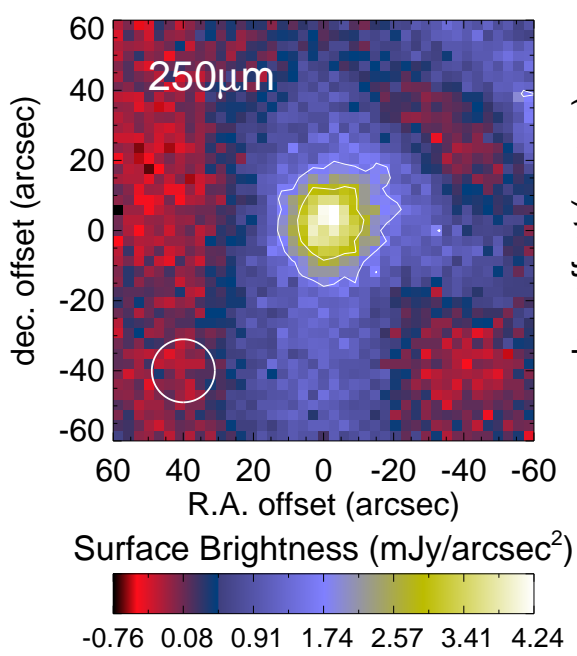

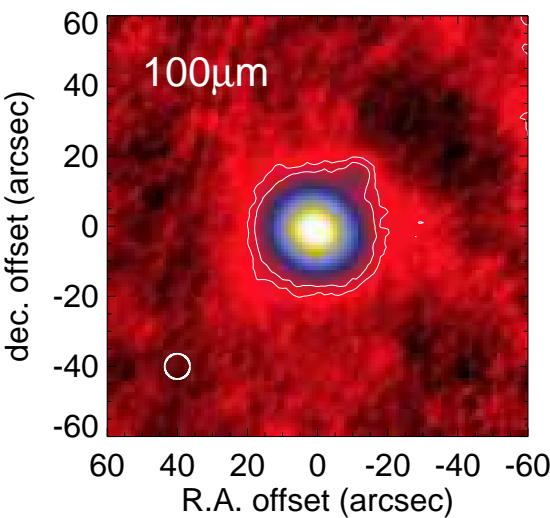

Surface Brightness (mJy/arcsec $\left.{ }^{2}\right)$
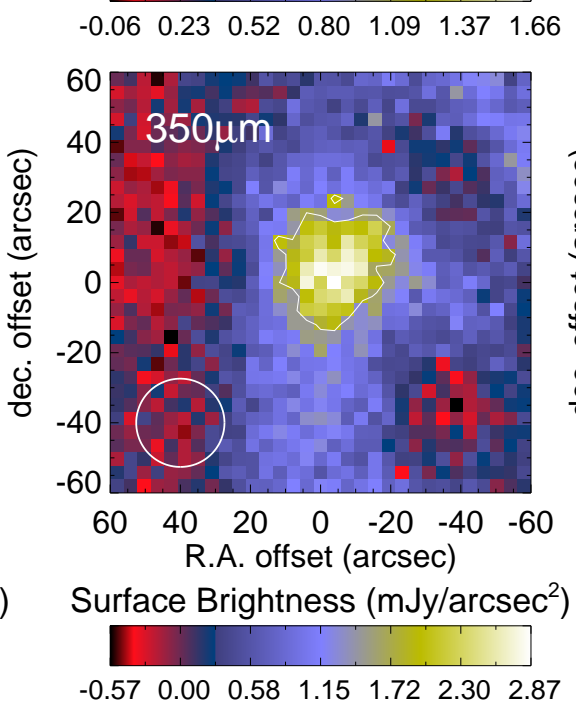

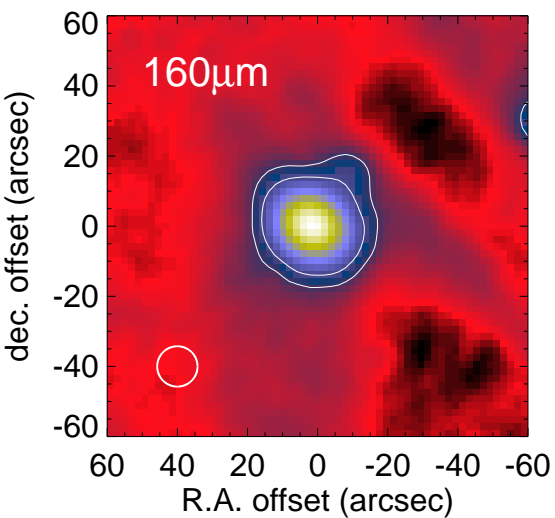

Surface Brightness (mJy/arcsec $\left.{ }^{2}\right)$

$\begin{array}{lllllll}-0.32 & 0.45 & 1.21 & 1.98 & 2.74 & 3.51 & 4.27\end{array}$

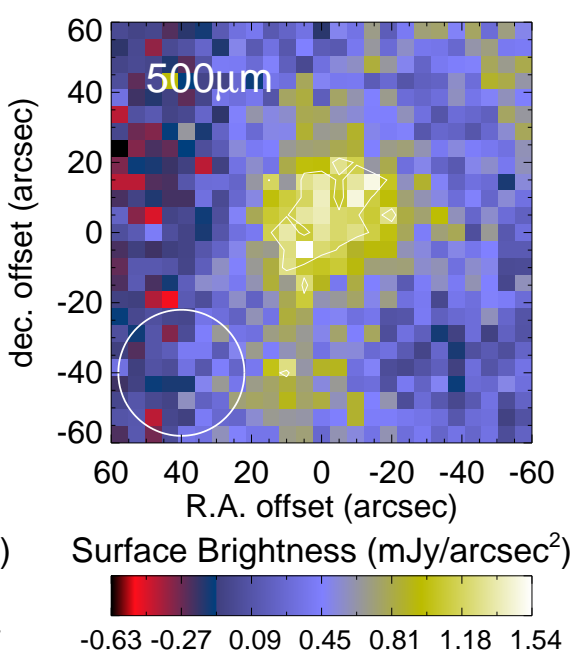

Figure 1. Far-infrared and submillimeter maps of HR 8799 from PACS and SPIRE. Different surface brightness scales are used for each map, and the pixel scales used are $1^{\prime \prime}$ pixels at 70 and $100 \mu \mathrm{m}, 2^{\prime \prime}$ pixels at $160 \mu \mathrm{m}$, and 3,4 and $5^{\prime \prime}$ pixels at 250,350 and $500 \mu \mathrm{m}$, respectively. These pixels are small for SPIRE data but the dithering used during these observations makes such fine sampling possible.

Table 3

Measured fluxes

\begin{tabular}{rccc}
\hline \hline $\begin{array}{c}\lambda \\
{[\mu \mathrm{m}]}\end{array}$ & $\begin{array}{c}F_{\text {aper }}{ }^{\mathrm{a}} \\
{[\mathrm{mJy}]}\end{array}$ & $\begin{array}{c}F_{\text {phot }} \\
{[\mathrm{mJy}]}\end{array}$ & $\begin{array}{c}F_{\text {disk }} \\
{[\mathrm{mJy}]}\end{array}$ \\
\hline 70 & $537 \pm 15$ & $6.85 \pm 0.07$ & $533 \pm 15$ \\
100 & $687 \pm 20$ & $3.39 \pm 0.04$ & $712 \pm 20$ \\
160 & $570 \pm 50$ & $1.35 \pm 0.01$ & $569 \pm 50$ \\
250 & $309 \pm 30$ & $0.550 \pm 0.006$ & $307 \pm 30$ \\
350 & $163 \pm 30$ & $0.276 \pm 0.003$ & $163 \pm 30$ \\
500 & $74 \pm 30$ & $0.135 \pm 0.002$ & $74 \pm 30$ \\
\multicolumn{4}{c}{ a BG source subtracted through PSF fitting. }
\end{tabular}

background. For example, a $30^{\prime \prime}$ aperture gives about $700 \mathrm{mJy}$, but a $40^{\prime \prime}$ aperture yields $760 \mathrm{mJy}$. Obviously, not all "estimates" are equally good. Given these uncertainties, we take the model flux of 570 mJy as our "best" $160 \mu \mathrm{m}$ value (as shown in Table 3), but assign an uncertainty of 50 mJy (i.e., a $S / N$ of 11 is achieved on the disk flux density). This value is higher than, but consistent with, the 539 mJy flux derived from Spitzer MIPS at the same wavelength (Su et al. 2009).

At SPIRE wavelengths, the background dominates the images so the photometry is less certain. The fluxes were estimated by subtracting smooth models such that the background looked even, and adopting conservative uncertainties. The Herschel photometry and uncertainties are presented in Table 3. The disk is well detected at 250 and $350 \mu \mathrm{m}$ ( $S / N$ of 10 and 5 , respectively), but has a flux detection of just $2.5 \sigma$ at $500 \mu \mathrm{m}$, where a significant part of the total detected flux density arises from the underlying cloud.

\subsection{Disk Size and Orientation}

To measure the disk size directly from the images, we did 2D Gaussian fits to the star+disk emission of HR 8799. We do not attempt to fit the background source simultaneously with the star and disk. The fits are first done to restricted areas around the target due to the increasing cirrus emission at longer wavelengths. After the full width half maximum and position angle of the fits is established, the models are extended to larger scales. The orientation of the best-fit Gaussians changes between the PACS and SPIRE data; it is likely that the background source (unresolved at these wavelengths from the HR 8799 disk), is impacting the orientation of the fit in these data. The sizes of the fitted Gaussians derived 


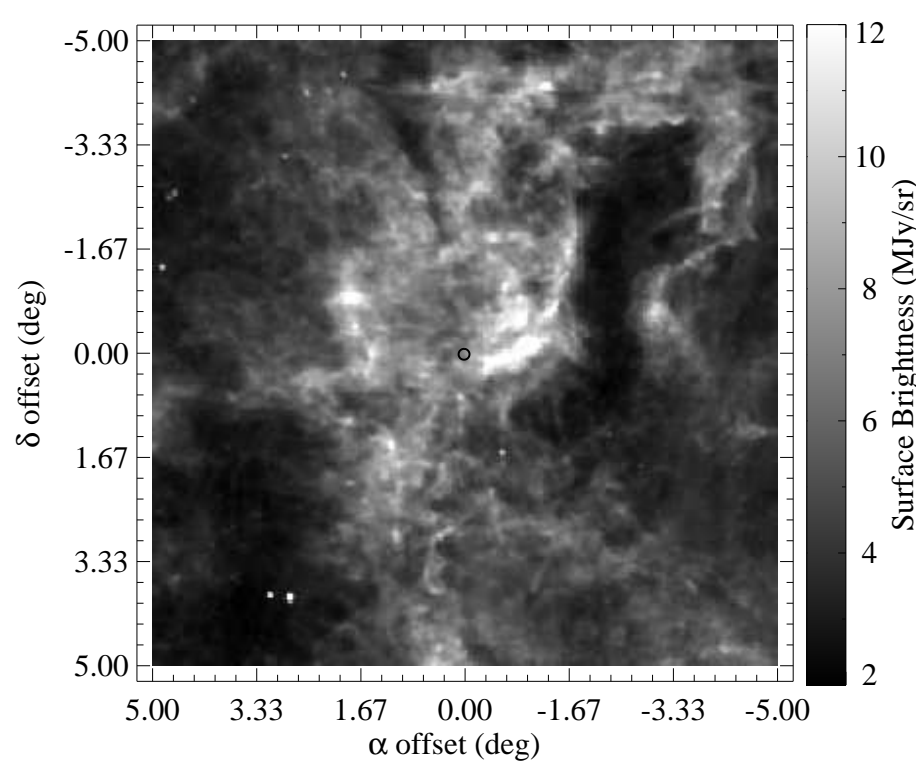

Figure 2. IRAS $100 \mu \mathrm{m}$ image of the region surrounding HR 8799 , marked at the field center by a circle.

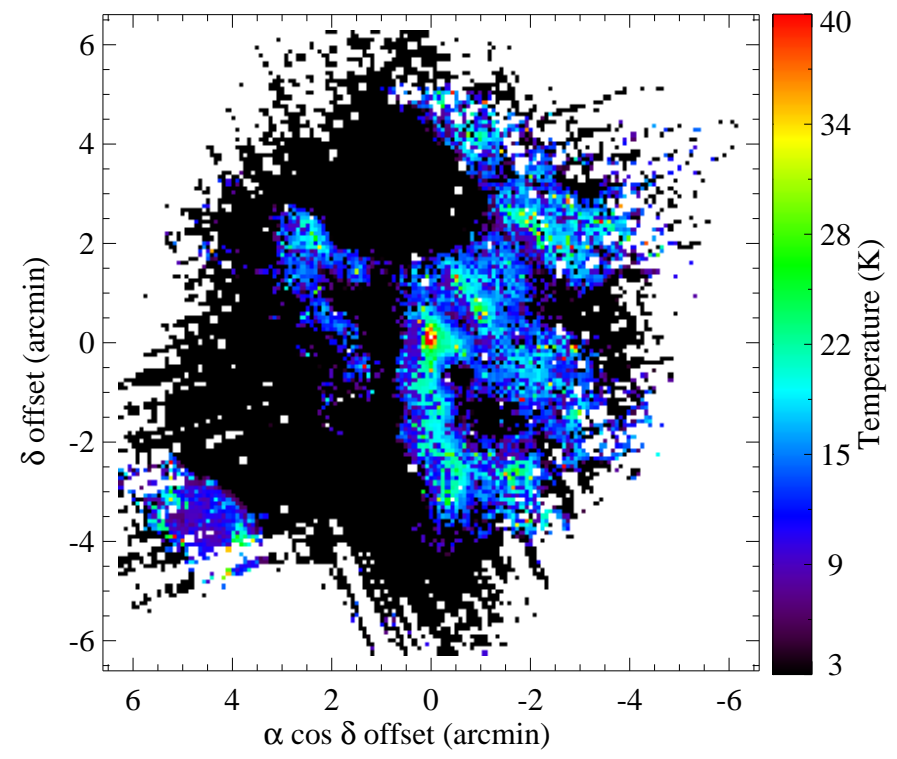

Figure 3. Dust temperature map derived from the SPIRE data, illustrating the cold large-scale emission from a background molecular cloud along the line of sight to the position of HR 8799 .

from the PACS maps are shown in Table 4. These fits provide consistent results for the 70 and $100 \mu \mathrm{m}$ data, both for the deconvolved size of the FWHM of the disk and its inclination and position angle.

\subsection{Spectral Energy Distribution}

Figure 4 shows the spectral energy distribution (SED) for HR 8799 using photometry from a wide range of sources. For the stellar component we fit PHOENIX models (Brott \& Hauschildt 2005) to optical and nearIR photometry (Svlvester et al. 1996; Perryman \& ESA 1997; Høg et al. 2000; Cutri et al. 2003), finding $T_{\text {eff }}=$

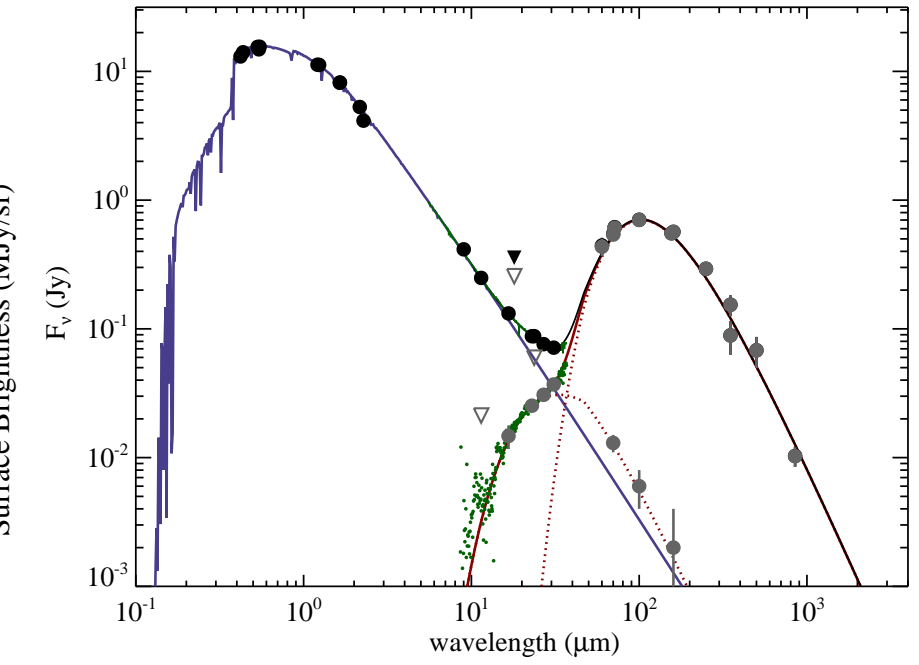

Figure 4. Star + disk SED for HR 8799, showing flux densities (dots) and upper limits (triangles). Shown are observed (black symbols) and star-subtracted fluxes (grey symbols), and the IRS spectrum (green dots). At long wavelengths, the gray dots cover the black dots completely, due to the declining flux density of the photosphere. At intermediate wavelengths, the star-subtracted flux densities are divided between the warm and cold disk components; therefore, there are two flux densities plotted for those wavelengths. The stellar photosphere model is shown as a blue line, the disk model as a red dotted line, and the star+disk spectrum as a black line.

$7380 \mathrm{~K}$ and $L_{\star}=5.4 L_{\odot}$, equivalent to a late-A or earlyF spectral type star, consistent with HR 8799's published spectral types (A5V, F0V, Gray \& Kaye 1999; Gray et al. 2003).

The Herschel photometry from 70-500 $\mu \mathrm{m}$ provides coverage over a wide range, and combined with previous data (Moshir \& et al. 1990; Williams \& Andrews 2006; Su et al. 2009; Ishihara et al. 2010; Patience et al. 2011), gives a fairly complete picture of the disk spectrum. To this photometry we have fitted a two-component modified blackbody model. The "modification" refers to two additional parameters, called $\lambda_{0}$ and $\beta$, with which a pure blackbody spectrum is modified beyond $\lambda_{0}$ by the multiplicative factor $\left(\lambda_{0} / \lambda\right)^{\beta}$. This factor attempts to account for inefficient emission by grains that are small relative to the wavelength of their emission. Therefore, $\lambda_{0}$ should in some way be representative of the grain size that dominates the emission spectrum. HR 8799 is also thought to have an unresolved inner warm disk component. Herschel photometry for this component is uncertain due to degeneracy with parameters for the outer disk, see $\$ 5$ for further discussion.

The best fit model shown in Figure 4 has temperatures of $153 \pm 15 \mathrm{~K}$ and $36 \pm 1 \mathrm{~K}$ for the two dust components, with $\lambda_{0}=47 \pm 30 \mu \mathrm{m}$ (upper limit $90 \mu \mathrm{m}$ ) and $\beta=$ $1.0 \pm 0.1$ common to both. Compared to most known debris disks, where $\lambda_{0}$ is usually $100 \mu \mathrm{m}$ or longer (e.g., Booth et al. 2013), the relatively short $\lambda_{0}$ here suggests that the disk spectrum is dominated by smaller grains than is typical in other debris disks. Given that HR 8799 is already known to be somewhat unusual due to its very extended disk that may comprise small, radiationdominated grains on eccentric orbits, is seems likely that the short $\lambda_{0}$ is somehow related to the halo. 
Table 4

2D Gaussian fits to star+disk emission in PACS maps

\begin{tabular}{|c|c|c|c|c|c|c|c|c|}
\hline \multirow{2}{*}{$\begin{array}{l}\text { Band } \\
(\mu \mathrm{m})\end{array}$} & \multirow{2}{*}{$\begin{array}{c}\text { beam } \\
\left({ }^{\prime \prime}\right)\end{array}$} & \multicolumn{2}{|c|}{ FWHMs } & \multicolumn{2}{|c|}{ Deconvolved } & \multirow{2}{*}{$\begin{array}{l}\text { Disk Size } \\
\quad(\mathrm{AU})\end{array}$} & \multirow{2}{*}{$\begin{array}{c}\text { Inclination }^{\mathrm{b}} \\
\left({ }^{\circ}\right)\end{array}$} & \multirow{2}{*}{$\begin{array}{c}\text { Position Angle } \\
\left({ }^{\circ} \mathrm{E} \text { of } \mathrm{N}\right)\end{array}$} \\
\hline & & ajor $\left({ }^{\prime \prime}\right)$ & minor $\left({ }^{\prime \prime}\right)$ & ajor $\left({ }^{\prime \prime}\right)$ & minor $\left({ }^{\prime \prime}\right)$ & & & \\
\hline 70 & & 00 & & & & & & \\
\hline 10 & & & & & & & & \\
\hline 160 & 11.36 & 4 & & & & & $33.4 \pm 6.5$ & 54.8 \\
\hline
\end{tabular}

${ }^{a}$ Adopting a distance of 39.9 pc for HR 8799 and assuming a circular disk.

b Calculated using deconvolved disk sizes.

\section{IMAGE MODELLING}

\subsection{Herschel}

Our goal here is to present a simple characterisation of the Herschel observations of HR 8799, not a detailed model that includes possible grain properties. We therefore model the data at each wavelength independently to arrive at a surface brightness profile for each wavelength. For the SPIRE data, which are strongly affected by the background level, we scale the PACS models to make the background level look smooth to derive disk+star flux densities.

The parameterized model is basically the same as used previously for modelling Herschel PACS observations (Kennedy et al. 2012a,b; Wyatt et al. 2012). Because we model each wavelength independently, however, the temperature profile is not needed and the model produces surface brightness images to compare with the data. The models are inspired by the Su et al. (2009) model, comprising three distinct components. The first is unresolved, which we simply add to the stellar flux when generating the model images. The other two components are called the "belt" and "halo", extending from $r_{1}$ to $r_{2}$ and $r_{2}$ to $r_{3}$. Each wavelength has a power-law change in surface brightness with radius for each component (i.e. $\left.\alpha_{\lambda, \text { comp }}\right)$. As found by Su et al. (2009), the belt component has a reasonably constant surface brightness, while the halo component drops steeply with radius.

The PACS models are created as three dimensional structures and then viewed with a specific geometry specified by the disk inclination and position angle to generate an image. These images are generated at high resolution and then convolved with the PACS beam (calibration observations of $\alpha$ Boo processed in the same way as the HR 8799 data), resulting in an image that can be directly compared with the science data. The overall brightness of each component and the power-law indices were varied to find a sufficiently good match to the data at each wavelength. We also varied $r_{1}, r_{2}$, and $r_{3}$, though these were required to be the same at all three wavelengths so the models are not completely wavelength independent.

A final component for the PACS models is the NE background source, which is simply added as a background point source whose location is the same at each wavelength, but whose brightness is allowed to vary with wavelength.

For SPIRE, we used a model that attempted to achieve an overall global fit to all data simultaneously rather than choose one of the individual PACS models. The resolution at these wavelengths is not sufficiently good, and the background level sufficiently high, that this choice is not important, given that these models were only used to provide estimates of the disk photometry.

The results of the modelling are shown in Figure 5. which shows the PACS data, each model, and the residuals after subtracting the model. While some residual structure remains due to variations in the background level, in each case there is no evidence for significant departures from azimuthal symmetry. We return to this topic in the Discussion section $(\S[6)$.

We found radii of $r_{1}=100 \mathrm{AU}, r_{2}=310 \mathrm{AU}$, and $r_{3}=2000 \mathrm{AU}$. The best fit power-law parameters and unresolved component fluxes are given in Table 5. We do not consider the contraints on the unresolved flux, the belt inner edge, and the belt power-law index to be very strong, particularly at $160 \mu \mathrm{m}$; the belt inner edge lies within the PACS beam so these parameters are all degenerate (see also Fig [6). At $160 \mu \mathrm{m}$, we have in fact fixed the unresolved flux and the belt and halo power-law indices to "sensible" values, by which we mean they do not lead to unusual conclusions about the radial temperature structure in the outer disk (see below). The outer disk radius is also poorly constrained and could continue to larger radii where the emission is here dominated by noise. The outer radius is considered representative to within a few hundred AU, but we note the caveat that we cannot be assured that all the flux has been captured in our maps, given the filtering and PSF effects (see $\S 3$ ). The power-law indices at 70 and $100 \mu \mathrm{m}$ for the halo are moderately well constrained (under the assumption that a power law is correct), with values of $\alpha_{\text {Halo }}=-4 \pm 0.3$ and $-3.5 \pm 0.5$ at 70 and $100 \mu \mathrm{m}$ respectively, with the uncertainties derived from the radial profiles fits, rather than the model images. The value of $13 \mathrm{mJy}$ for the unresolved component at $70 \mu \mathrm{m}$ agrees reasonably well with the predicted value of about $18 \mathrm{mJy}$ if this component is a pure blackbody (Su et al. 2009). The values of 6 and $2 \mathrm{mJy}$ at 100 and $160 \mu \mathrm{m}$ are less certain due to poorer resolution, but these are less than expected for a pure blackbody based on the $13 \mathrm{mJy}$ value at $70 \mu \mathrm{m}$. However, given that all three lie along a line of $F_{\nu} \propto \lambda^{-2}$, and that this dependence is steeper than expected for a 150 $\mathrm{K}$ blackbody, it seems likely that the emission spectrum of the warm component is not a pure blackbody. Such a conclusion would be unsurprising given that debris disk spectra are commonly seen to be steeper than a blackbody in the far-IR. That the turn-over wavelength $\left(\lambda_{0}\right)$ is shorter than usually seen may be indicating relatively small grains or a relatively steep size distribution. We discuss this further in $\S 6.5$.

The disk inclination is $26^{\circ}$, for which we consider the uncertainty to be about $3^{\circ}$. The results from image modeling are completely consistent with the inclination based on 2D Gaussian fits to the data, and so do not appear to be strongly model dependent. To account for correlated 

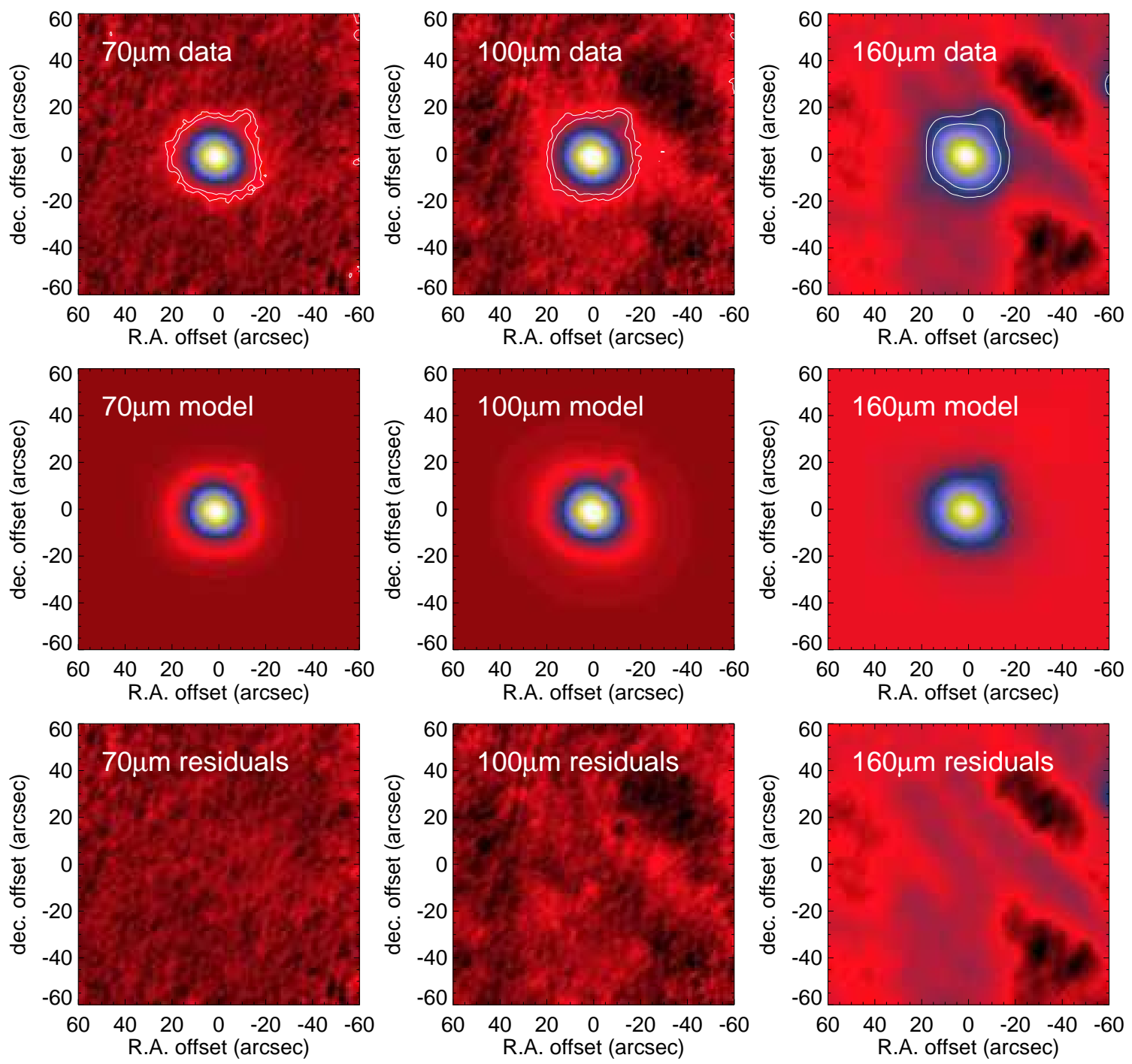

Figure 5. Results of PACS data models, showing the PACS data, model for each wavelength and the residual.

noise in the images, the uncertainty in the quantities derived from the images - inclination and position angle have been multiplied by 3.6 based on (Fruchter \& Hook 2002, see Kennedy et al. (2012a) for more details.). To estimate this uncertainty formally would be difficult as many model parameters would need to be marginalized over. To check that the estimate of a few degrees is sensible, we fitted 2D Gaussians to a star-subtracted image to see how the fit quality varied, with the same conclusion that the uncertainty is a few degrees. The position angle of the disk is $62 \pm 3^{\circ}$ East of North.

As a check on the aperture photometry in 4.1 , the total model fluxes, including the star but not the background source, are summarized in Table 6 (see photometry discussion above regarding the $160 \mu \mathrm{m}$ flux). Splitting these into contributions from the belt and halo components is also informative, given that the conclusion from the SED was that small grains dominate the overall spectrum. These component fluxes are also shown in Table 6. Therefore, the halo component contributes a significant amount of the flux at all Herschel wavelengths and dominates beyond $160 \mu \mathrm{m}$. If the halo comprises
Table 5

Wavelength-dependent model parameters.

\begin{tabular}{|c|c|c|c|c|}
\hline$\lambda[\mu \mathrm{m}]$ & $F_{\text {unres }}$ & {$[\mathrm{mJy}]$} & $\alpha_{\text {Belt }}$ & $\alpha_{\text {Halo }}$ \\
\hline 70 & & 13 & -1.0 & -4.0 \\
\hline 100 & & 6 & -0.9 & -3.5 \\
\hline 160 & & 2 & -1.0 & -3.0 \\
\hline
\end{tabular}

Note. - The parameters are poorly constrained at $160 \mu \mathrm{m}$ because the inner part of the disk is poorly resolved ( $F_{\text {unres }}, \alpha_{\text {belt }}$, and $r_{1}$ are degenerate) and the background is much higher $\left(\alpha_{\text {halo }}\right.$ is uncertain). The values at $160 \mu \mathrm{m}$ were therefore chosen to agree with the conclusions based on 70-100 $\mu \mathrm{m}$ data (see text).

mostly small grains then the relatively short $\lambda_{0}$ value is not surprising.

Because the HR 8799 disk appears azimuthally symmetric, we now consider the radial profiles of both the observed and modelled emission. These are shown in Figure 6, where it is again clear that the model repro- 
Table 6

Relative Flux Contributions from the Belt and Halo.

\begin{tabular}{rccc}
\hline \hline$\lambda[\mu \mathrm{m}]$ & $F_{\text {model }}[\mathrm{mJy}]$ & $F_{\text {belt }}[\mathrm{mJy}]$ & $F_{\text {halo }}[\mathrm{mJy}]$ \\
\hline 70 & 550 & 304 & 215 \\
100 & 700 & 339 & 325 \\
160 & 570 & 236 & 298 \\
\hline
\end{tabular}

duces the data well. The break in the surface brightness profile around $10^{\prime \prime}$ is apparent, and justifies use of the two-component belt+halo model for the outer disk. As noted above, the steepness with which the belt surface brightness drops with radius is somewhat degenerate with the unresolved flux from the warm component, particularly at $160 \mu \mathrm{m}$. While the model drops below the observed profile at this wavelength, it leaves a smooth background so is satisfactory. It is also clear that the data constrain the surface brightness profile fairly well out to $20-30^{\prime \prime}$ (800-1200 AU) while the emission is detected out to $2000 \mathrm{AU}$, particularly at 100 and $160 \mu \mathrm{m}$.

\subsection{Spitzer MIPS $24 \mu m$}

While the PACS data are reasonably well resolved, they do not provide particularly wide coverage in terms of wavelength. Therefore, we now consider the disk as resolved at $24 \mu \mathrm{m}$ by Spitzer (Su et al. 2009). Our starting point is a star-subtracted $24 \mu \mathrm{m}$ image processed as described in that paper. The extended disk emission in this image has relatively low $\mathrm{S} / \mathrm{N}$, so we only consider the radial profile, which is shown in the top panel of Figure 7.

To derive the profile of the extended disk component we follow Su et al. (2009) and subtract a point source model scaled to the peak emission at the stellar position (here a $3.5^{\prime \prime}$ boxcar-smoothed STinyTim model) to remove the contribution from the unresolved warm disk emission. This procedure is justified because the total belt+halo emission is at most $\sim 5 \%$ of the total $24 \mu \mathrm{m}$ emission, of which only a small fraction is expected to arise from the stellar position. The resulting belt+halo radial profile is shown in the bottom panel of Figure 7 .

As described by Su et al. (2009), the disk is seen to be extended. The significance of the signal is weak near the stellar position due to only being slightly brighter than the point source model, and weak at large radii due to low $\mathrm{S} / \mathrm{N}$. However, the signal is strong from $5-50^{\prime \prime}$, where it decays monotonically. A small difference here is that we find that the surface brightness is significantly above zero to slightly larger distances (about 60" rather than $\left.50^{\prime \prime}\right)$. The overall trend is similar, in that the surface brightness decays from about $10^{-5} \mathrm{Jy} \operatorname{arcsec}^{-2}$ at a few arcseconds radius to a few $10^{-7} \mathrm{Jy} \mathrm{arcsec}^{-2}$ at $50^{\prime \prime}$ radius.

To derive the underlying surface brightness distribution, we convolved a two-power-law model (i.e. belt+halo) with the STinyTim PSF to find the best fitting via $\chi^{2}$ minimisation. During this process we fixed the belt and halo radii to those found from the PACS data (i.e. 100, 310, and $2000 \mathrm{AU}$ ). The best fitting model is shown in the bottom panel of Figure 7 While it is clear that the slope of the halo component is fairly well constrained, the inner component is not due to the large surface brightness uncertainty at small radii. The power-law slopes are $-3.3 \pm 0.1$ and $-1 \pm 0.06$ for the

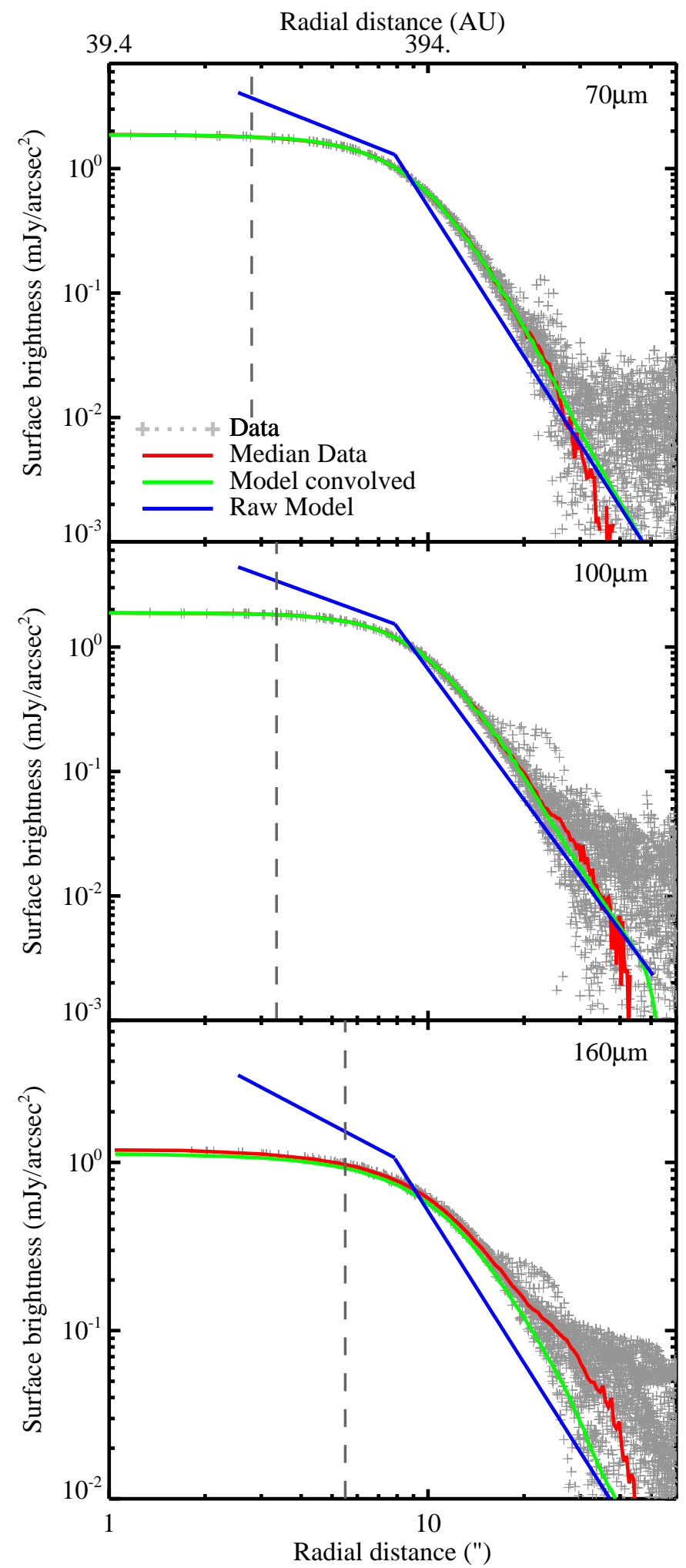

Figure 6. HR 8799 radial profiles at the three PACS wavelengths. Each panel shows the raw surface brightness values for all pixels within $60^{\prime \prime}$ of the star (dots) and their median (red), the median convolved model surface brightness (green), and the raw unconvolved profile (blue). All profiles are generated using the image models, and assume a disk inclination of $25.75^{\circ}$ and PA of $63.73^{\circ}$. The dashed grey lines represent the half-width at half-maximum of the beam. 

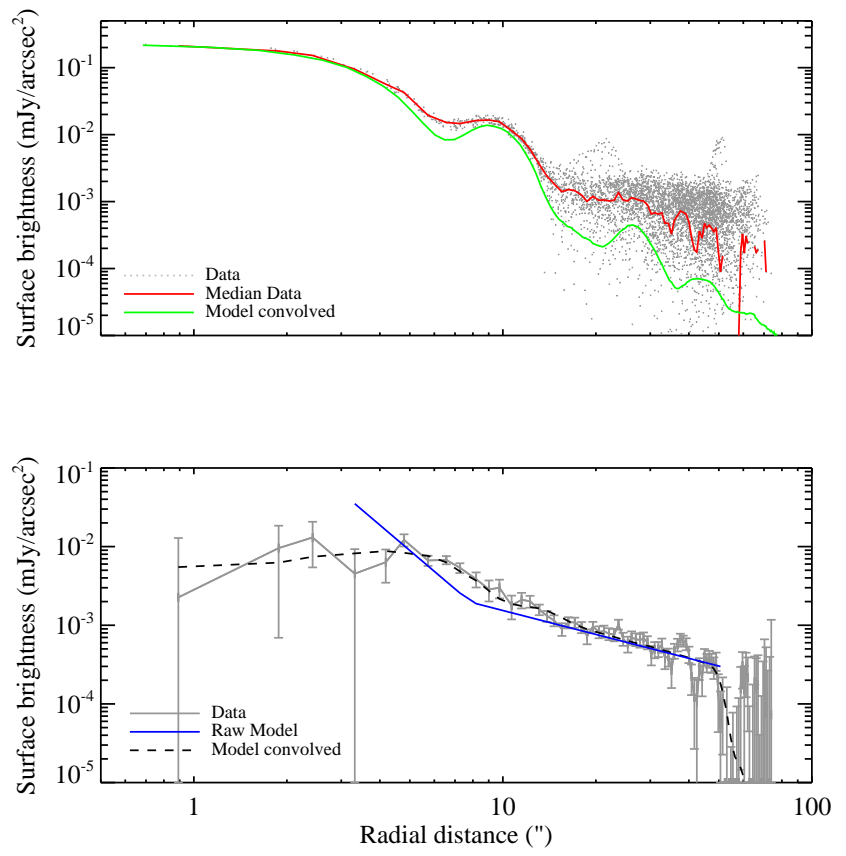

Figure 7. Top: Radial profile at $24 \mu \mathrm{m}$. Bottom: Star-subtracted outer disk profile and surface brightness model fit. Aside from a difference in units, panels are directly comparable with Figure 6 of Su et al. (2009).

belt and the halo, respectively, with the uncertainties derived from the diagonal covariance matrix elements. The uncertainties are probably somewhat larger due to systematic uncertainties, such as the correctness of the point source model. However, as we show below, the observed profile is interesting when compared with the Herschel data.

\subsection{Combined radial profiles}

Though the surface brightness profiles highlight the radial structure well, the unconvolved model profiles can be taken one step further to show the disk emission spectrum as a function of radius, as shown in Figure 8. These combine the MIPS and PACS data described above. At each radius we have divided by a colour correction calculated using a blackbody given by the $70-100 \mu \mathrm{m}$ flux ratio for the 70,100 and $160 \mu \mathrm{m}$ fluxes, and a correction using the $24-70 \mu \mathrm{m}$ correction for the $24 \mu \mathrm{m}$ fluxes. This correction is only a few percent in the PACS bands and has a maximum of 1.3 at $300 \mathrm{AU}(39 \mathrm{~K})$ for MIPS. As noted below the temperatures used for these colour corrections are uncertain, but as they are small, different temperatures will not significantly change our conclusions.

For all radii Figure 8 shows that the disk spectrum at the PACS wavelengths of 70, 100 and $160 \mu \mathrm{m}$ is well described by a blackbody. However, this agreement is contrived at $160 \mu \mathrm{m}$ due to uncertain model parameters. Therefore, we have only used the $70+100 \mu \mathrm{m}$ data to derive the blackbody temperature at each wavelength. We also derive a blackbody temperature for the $24+70 \mu \mathrm{m}$ colour. While we would ideally fit two blackbodies simultaneously, with only three wavelength measurements (ignoring $160 \mu \mathrm{m}$ ) at each radius the temperatures are degenerate so we take these independent values as repre-

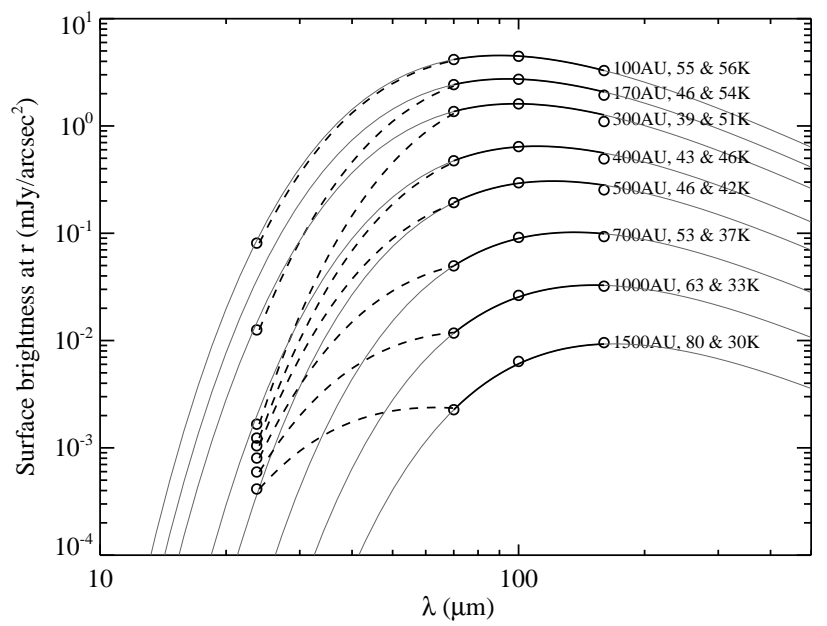

Figure 8. Disk emission profile at different radii. A blackbody fit to the $70-100 \mu \mathrm{m}$ data is shown at each radius (solid lines) as well as a blackbody fit to the $24-70 \mu \mathrm{m}$ data (dotted lines), with the two temperatures given at each radius. With only three reliable wavelength points at each radius the temperatures are degenerate, so here we just show independent fits to each pair of 24-70 and $70-100 \mu \mathrm{m}$.

sentative.

The main point is that the derived temperatures are significantly different for $24+70$ versus $70+100 \mu \mathrm{m}$. At the innermost distances the agreement is reasonable given the uncertainty in the MIPS profile, but not beyond $\sim 500 \mathrm{AU}$. The relatively flat power-law decay of the MIPS surface brightness profile means that as the disk temperature as derived from PACS drops, the observed MIPS surface brightness becomes increasing high compared to the expected level. This discrepancy might be questioned at the outermost radii, where small differences in the assumed background level could cause the MIPS profile to be lower (e.g., zero at $50^{\prime \prime}$ ). However, at $20^{\prime \prime}$ (800 AU) the discrepancy is harder to explain away. The expected $37 \mathrm{~K}$ disk temperature predicts a $24 \mu \mathrm{m}$ surface brightness of about $10^{-5} \mathrm{mJy} \operatorname{arcsec}^{-2}$, but the observed level is two orders of magnitude higher. The radial profiles from $\mathrm{Su}$ et al. (2009) are slightly fainter than ours, but the discrepancy is still far larger than expected.

To achieve even a rough agreement of the $24 \mu \mathrm{m}$ radial profile with the levels predicted by the PACS $70-100 \mu \mathrm{m}$ temperatures requires subtracting a DC level of $7 \times 10^{-4}$ mJy $\operatorname{arcsec}^{-2}$ from the MIPS image. The median and mean pixel levels are $-1 \times 10^{-4}$ and $-6 \times 10^{-5} \mathrm{mJy}$ $\operatorname{arcsec}^{-2}$ respectively, with a standard deviation of $10^{-3}$ mJy $\operatorname{arcsec}^{-2}$. Therefore, such a subtraction is unjustified because it shifts the mean value in the MIPS image by nearly $1 \sigma$. Further, such a subtraction causes the median radial profile to become negative beyond $30^{\prime \prime}$ (i.e. in a region where Figure 7 shows it is still clearly decreasing with radius).

Therefore, while there is some uncertainty in the absolute level, it seems that the levels predicted from the temperatures derived from the PACS data and the levels seen by MIPS are in clear disagreement, most significantly at large radii ( $\gtrsim 500 \mathrm{AU})$. The same conclusion would be reached using the radial profiles derived by $\mathrm{Su}$ et al. (2009), where there is still significant disk emission at 
$30^{\prime \prime}$ (a few $10^{-4} \mathrm{mJy} \operatorname{arcsec}^{-2}$ at $1200 \mathrm{AU}$ ), where the PACS-predicted level is less than $10^{-5} \mathrm{mJy} \operatorname{arcsec}^{-2}$, as indicated in Figure 8. Indeed, their favoured model had a halo component that was warmer than the belt component, despite the larger distance, which was attributed to the halo comprising smaller grains and seems consistent with the $24+70 \mu \mathrm{m}$ temperature profile derived here.

Alternatively, the PACS 70 or $100 \mu \mathrm{m}$ profiles would need to be at least a factor of two in error at these distances to allow a single temperature consistent with the MIPS data. However, the good agreement between the data and model in Figure 6 show that such a difference is very unlikely.

\section{DISCUSSION}

\subsection{Evidence for Azimuthal Structure}

Visual inspection of the data reveals no apparent asymmetries in the emission from the HR 8799 disk, excepting the compact northwest feature. This is in contrast to recent findings in $350 \mu \mathrm{m}$ maps from the CSO (Patience et al. 2011). Non-axisymmetric structure was observed, which was interpreted as evidence of particles trapped in a 2:1 resonance with the outer planet. We note that our resolution at $350 \mu \mathrm{m}$ is poorer than that of the CSO by an approximate factor of 2 . Other than those ground-based data, no asymmetries have been seen in the disk. Hughes et al. (2011) attribute the asymmetries in interferometric maps from the SMA to noise features in low signal-to-noise data. Our data are consistent with this picture. We note that strong asymmetries are not typically expected at far-infrared wavelengths, but rather models suggest that the strongest asymmetries will be observable in the submillimeter where the data sample the larger grains in the disk which are more likely to be localized in the planetesimal belts and trapped in resonances.

To place a limit on the brightness of a clump which would have been detected, we take the $5 \sigma$ limit at $70 \mu \mathrm{m}$ as an example. The rms at $70 \mu \mathrm{m}$ is $0.34 \mathrm{mJy}$ beam $^{-1}$, and to see a believable substructure in the disk would have required a peak flux of $1.7 \mathrm{mJy}$. In contrast, the symmetrically distributed disk emission has a $70 \mu \mathrm{m}$ flux of roughly $530 \mathrm{mJy}$. Therefore, a "discrete" peak in the disk of flux density $\sim 0.3 \%$ of the symmetrical emission should have been detectable. The $70 \mu \mathrm{m}$ beam has a scale of $220 \mathrm{AU}$ at the distance of HR 8799, so even unresolved point sources could cover a significant fraction of the inner disk. Our ability to place limits on our sensitivity to still larger resolved clumps in the disk is degraded by the large filter scale applied during data reduction, a scale required to accurately recover the largest scales of the halo.

\subsection{Implications for Planet Formation Scenarios}

The fact that no significant azimuthal structures were observed in the Herschel images of the HR 8799 disk is significant because a planet formation scenario in which planets form close to the star and then migrate outward to their present positions would have favored the trapping of planetesimals into resonances (Wvatt 2003). The resulting resonant structure would result in the planetesimal distribution having a clumpy structure, and observations of the level and morphology of that clumpiness would be indicative of the planets' migration rate and therefore of the mechanism which caused the migration (e.g., Crida et al. 2009). Dynamical stability studies have suggested that the HR 8799 planets could themselves be in resonance (Fabrvckv \& Murrav-Clav 2010; Reidemeister et al. 2009; Goździewski \& Migaszewski 2009, 2013). This does not de facto imply migration has occurred in the system, since the formation of planets in situ by gravitational instability (e.g., Dodson-Robinson \& Bodenheimer 2010) can also result in resonant configurations.

Comparison with observations of the disk is complicated by the fact that we observe the distribution of dust, and not of planetesimals. Observations at wavelengths $>200 \mu \mathrm{m}$ are sensitive to dust that is large enough to trace the planetesimal distribution. However, shorter wavelength observations are sensitive to small dust sizes that can have an axisymmetric distribution even if the planetesimals are themselves in resonance (Wvatt 2006). Given the low resolution of our SPIRE data, and the short wavelength of the higher resolution PACS images, we cannot set strong constraints on the resonant structure of the planetesimal belt in this system from our Herschel observations, though this is implied by the previous publication of an asymmetry in the disk at $350 \mu \mathrm{m}$ (Patience et al. 2011). If such asymmetric structures are present, then they should be most readily detectable by ALMA.

\subsection{Constraints on System Inclination and Orientation}

From our disk model based on well-resolved images, the inclination of the disk is $26 \pm 3^{\circ}$. Given the azimuthal symmetry we observe, it is unlikely that the disk is inclined at significantly $>25^{\circ}$. Similarly, the Su et al. (2009) Spitzer data also ruled out inclinations larger than $\sim 25^{\circ}$. Inclinations higher than $45^{\circ}$ are not supported by the disk images, assuming a circular disk geometry inherent in the disk system.

Estimates of the stellar inclination from astroseismology suggest $i>40^{\circ}$ (Wright et al. 2011), although a value as low as $35^{\circ}$ is not ruled out by their work (see their Figure 3), but these values are inconsistent with the modeling of Reidemeister et al. (2009) which suggest a near face-on inclination of $13-30^{\circ}$. This is consistent with the orbit modeling for the detected planets (Marois et al. 2008; Fabrvckv \& Murrav-Clay 2010; Lafrenière et al. 2009; (Soummer et al. 2011), and with estimates from the debris disk modeling presented in Su et al. (2009).

The suggestion that the entire HR 8799 system may be coplanar is consistent with the results of Watson et al. (2011) and Greaves et al. (2013), which includes HR 8799 , who found common inclinations between a significant number of stars for which the stellar and disk inclinations had been independently measured. In addition, the recent work by Goździewski \& Migaszewski (2013) on the astrometry of the HR 8799 system reports an inclination of $\sim 24^{\circ}$ for the planetary orbits and measures the longitude of the ascending node to be $\sim 64^{\circ}$. The disk position angle $\left(62 \pm 3^{\circ}\right)$ is measuring the disk longitude of ascending node, so if the planets are coplanar with the disk, we should expect both values to agree within uncertainties, which they clearly do. It is therefore likely that the entire HR 8799 star-disk-planet system is coplanar. 


\subsection{Constraints on the Eccentricity of the Disk}

Any eccentricity in the disk is degenerate with the inclination; therefore, the best constraint on nonaxisymmetry comes from the fact that one side is not seen to be brighter than the other. Conservatively, the PACS $70 \mu \mathrm{m}$ observations are sensitive to a $\leq 1 \%$ brightness asymmetry, which corresponds to roughly a $2 \%$ difference in dust radial distances from one side of the disk to the other (assuming a linear dependence of flux density on temperature). Such an asymmetry would arise if planetary eccentricities caused the disk to become offset from the star due to secular perturbations (i.e as seen for the debris ring around Fomalhaut, Kalas et al. 2005). Fomalhaut shows a clear asymmetry from PACS images of similar S/N with an eccentricity of 0.1 (Acke et al. 2012). The inner edge of the cold belt of HR 8799's disk is at a similar radial distance as that of Fomalhaut; therefore, the disk eccentricity expected for HR 8799 must be comparable to, or below, that level.

\subsection{Preponderance of Small Grains}

The relatively short value of $\lambda_{0}<90 \mu \mathrm{m}$ for the outer component (belt+halo) from the SED (44.3) suggests that the emission from this component is dominated by grains smaller than this wavelength (or equivalently has a relatively steep size distribution overall). It seems likely that this dominance is related to the presence of the outer halo, though interestingly the disks around Fomalhaut, Vega and $\beta$ Pictoris, the best studied systems with haloes, have $\lambda_{0}$ from $150-250 \mu \mathrm{m}$. Clearly then, the existence of a detectable halo does not require a short $\lambda_{0}$. The question may therefore be one of their relative brightness; a brighter halo weights the overall size distribution to small sizes. One way to increase the halo brightness is with a recently increased level of dust due to collisions, this increase both injects more particles into the bound (high eccentricity) part of the halo, as well as the unbound (para/hyper-bolic) part. Little work has been done to compare halo models with those observed, most notably Müller et al. (2010) found that the halo around Vega could be explained with a steady-state halo model, in contrast to the original suggestion that it was due to a recent event (Su et al. 2005). The much shorter value of $\lambda_{0}$ seen for HR 8799 suggests that a similar analysis should be done, with a particular focus on the possible origins of an overabundance of small $(<50 \mu \mathrm{m})$ grains compared to the Vega disk.

Similarly, the existence of $24 \mu \mathrm{m}$ emission at larger radii than expected (Fig. 7) may be related to the presence of small grains. However, in this case the reason is unclear. A possible cause is that grains in the halo have more than one composition, thus resulting in more than one temperature at a single radial distance (although the narrow SED would seem to rule this out). Another potential reason is that HR 8799 is passing through an overdense part of the ISM, and that at least some of the observed SED actually comes from heated ISM dust rather than circumstellar dust. There is however no evidence in the images that HR 8799 is strongly perturbing the ISM around it, as has been observed for the nearby star $\delta$ Velorum (Gáspár et al. 2008).

The temperature of the blackbody fit implies a disc radius of $140 \mathrm{AU}$, which lies within the true radial distri- bution of the dust. This is surprising as the luminosity of HR $8799\left(5.4 L_{\odot}\right)$ results in a low blowout grain size meaning that we would expect there to be plenty of small grains in the belt itself, and these would be much hotter than blackbody (Booth et al. 2013). Such an inconsistency is not unheard of; for instance, Löhne et al. (2012) found that, for HD 207129, the dominant grain size was ten times higher than the blowout limit. The lack of small grains in the belt does also place extra emphasis on the dust in the halo being the dominant component to the SED.

As discussed in $\S 6.1$, based on the modeled symmetric disk, an unresolved clump of flux density $<1 \%$ of the total flux at $70 \mu \mathrm{m}$ would have been detectable. The smoothness could indicate that the halo is populated with bound high eccentricity small grains, as has been suggested for Vega based on Herschel observations (Sibthorpe et al. 2010), since such grains would have an axisymmetric distribution regardless of the resonant structure of the planetesimal belt (Wyatt 2006). However, a population of unbound grains could also be axisymmetric, and moreover the evidence for clumping in the parent planetesimal population is limited.

\section{SUMMARY}

We have imaged the HR 8799 debris disk with PACS and SPIRE on Herschel and modeled resolved imaging at 70, 100 and $160 \mu \mathrm{m}$. Based on the SED, we find evidence of two components, a warm unresolved component of $153 \pm 15 \mathrm{~K}$ and a cold, resolved component of $36 \pm 1$ $\mathrm{K}$. Imaging modeling reveals two distinct components of cold dust, as first identified by Su et al. (2009). We find evidence of a cold planetesimal belt, a "Kuiper Belt analogue", extending from 100-310 AU, and a more extended halo component from 310 - 2000 AU. The radial profiles of the dust emission show a clear break at $310 \mathrm{AU}$ in the power-law slopes, from shallower slopes of -0.9 to -1.0 for the planetesimal belt to steep slopes of -3.0 to -4.0 for the halo (depending on wavelength).

The radius inferred from the blackbody fit to the cold component of the SED is $140 \mathrm{AU}$, well within the planetesimal belt of HR 8799's disk. Therefore, unlike many other disks in which the grains are found at considerably larger radii than their blackbody fits would predict (Booth et al. 2013; Rodriguez \& Zuckerman 2012), in HR 8799 the grains appear to be very well fit as blackbodies.

The fluxes from the planetesimal belt and halo are comparable. The SED indicates a steepening of the spectrum at $\lambda_{0}=47 \pm 30 \mu \mathrm{m}$, with an upper limit of $90 \mu \mathrm{m}$. Taken as a representative of the grain size that dominates the emission spectrum, it therefore appears that HR 8799 is populated by smaller grains than other A star disks with haloes (e.g., Vega, $\beta$ Pic and Fomalhaut), as well as A star disks in general (Booth et al. 2013).

The combined radial profiles of our Herschel data and Spitzer $24 \mu \mathrm{m}$ data (Su et al. 2009) show that the derived temperatures are significantly different for $24+70$ $\mu \mathrm{m}$ versus $70+100 \mu \mathrm{m}$ beyond $500 \mathrm{AU}$ (i.e., in the halo). Therefore, while the PACS data imply that the halo is colder than the planetesimal disk (temperature decreasing with radial distance), the $24+70 \mu \mathrm{m}$ data suggest that the halo is warmer, consistent with the conclusions of Su et al. (2009). This could mean there are poten- 
tially two distinct populations of dust grains (sizes or compositions) in the halo and reinforces the importance of obtaining resolved imaging at multiple wavelengths to properly understand the interior physical structure of disks.

We constrain the inclination of the disk to be $26 \pm 3^{\circ}$ and the position angle to be $62 \pm 3^{\circ}$. These values are determined through image modeling and also through 2D Gaussian fits to the disk emission at all three PACS wavelengths. This value of the inclination is consistent with constraints from planetary modeling in the system as well as with the inclination of the star itself, suggesting that the system has strong alignment between all three components: star, planets and disk. The agreement between the disk position angle and the longitude of ascending nodes of the planets establishes that the system is coplanar.

There is no evidence of asymmetric structure in the HR 8799 disk. The symmetric models fit the disk so well that we estimate that, if substructure is present, individual unresolved clumps could be no brighter than $1 \%$ of the total flux in the symmetric distribution of emission. Based on this limit on brightness asymmetry in the disk emission, and comparable constraint from the similarity in the disk radial separation of HR 8799 with Fomalhaut, we estimate that the disk eccentricity must be $<0.1$.

We gratefully acknowledge the thorough report provided by our referee. BCM, MB and $\mathrm{HBF}$ acknowledge the support of a Discovery Grant and a Discovery Accelerator Supplement from the Natural Science and Engineering Council (NSERC) of Canada. MCW and GK are grateful for support from the European Union through ERC grant number 279973. A portion of this work was performed under the auspices of the U.S. Department of Energy by Lawrence Livermore National Laboratory under Contract DE-AC52-07NA27344.

\section{REFERENCES}

Acke, B., Min, M., Dominik, C., et al. 2012, A\&A, 540, A125

Backman, D., Marengo, M., Stapelfeldt, K., et al. 2009, ApJ, 690, 1522

Booth, M., Kennedy, G., Sibthorpe, B., et al. 2013, MNRAS, 428, 1263

Brott, I. \& Hauschildt, P. H. 2005, in ESA Special Publication, Vol. 576, The Three-Dimensional Universe with Gaia, ed. C. Turon, K. S. O'Flaherty, \& M. A. C. Perryman, 565

Bowler, B. P., Liu, M. C., Dupuy, T. J., \& Cushing, M. C. 2010, ApJ, 723, 850

Chambers, J. E. 2001, Icarus, 152, 205

Chiang, E., Kite, E., Kalas, P., Graham, J. R., \& Clampin, M. 2009, ApJ, 693, 734

Crida, A., Masset, F., \& Morbidelli, A. 2009, ApJ, 705, L148 x

Cutri, R. M. et al. 2003, 2MASS All Sky Catalog of point sources.

Dodson-Robinson, S. E., \& Bodenheimer, P. 2010, Icarus, 207, 491

Fabrycky, D. C., \& Murray-Clay, R. A. 2010, ApJ, 710, 1408

Fruchter, A.S., \& Hook, R.N. 2002, PASP, 114, 144

Gáspár, A., Su, K. Y. L., Rieke, G. H., et al. 2008, ApJ, 672, 974

Goździewski, K., \& Migaszewski, C. 2009, MNRAS, 397, L16

Goździewski, K., \& Migaszewski, C. 2013, MNRAS, submitted

Gray, R. O., Corbally, C. J., Garrison, R. F., McFadden, M. T., \& Robinson, P. E. 2003, AJ, 126, 2048

Gray, R. O., \& Kaye, A. B. 1999, AJ, 118, 2993

Greaves, J. et al. 2013, MNRAS, submitted

Greaves, J. S., Holland, W. S., Wyatt, M. C., et al. 2005, ApJ, 619, L187
Greaves, J. S., Holland, W. S., Moriarty-Schieven, G., et al. 1998, ApJ, 506, L133

Griffin, M. J., Abergel, A., Abreu, A., et al. 2010, A\&A, 518, L3

Høg, E., Fabricius, C., Makarov, V. V., Urban, S., Corbin, T., Wycoff, G., Bastian, U., Schwekendiek, P., \& Wicenec, A. 2000, A\&A, 355, L27

Holland, W. S., Greaves, J. S., Zuckerman, B., et al. 1998, Nature, 392, 788

Hughes, A. M., Wilner, D. J., Andrews, S. M., et al. 2011, ApJ, 740,38

Hughes, A. M., Wilner, D. J., Mason, B., et al. 2012, ApJ, 750, 82 Ishihara, D. et al. 2010, A\&A, 514, A1

Kalas, P., Graham, J. R., Fitzgerald, M. P., \& Clampin, M. 2013, ApJ, 775, 56

Kalas, P., Graham, J. R., \& Clampin, M. 2005, Nature, 435, 1067

Kalas, P., Graham, J. R., Chiang, E., et al. 2008, Science, 322, 1345

Kennedy, G. M., Wyatt, M. C., Sibthorpe, B., Duchêne, G., Kalas, P., Matthews, B. C., Greaves, J. S., Su, K. Y. L., \& Fitzgerald, M. P. 2012a, MNRAS, 421, 2264

Kennedy, G. M., Wyatt, M. C., Sibthorpe, B., Phillips, N. M., Matthews, B. C., \& Greaves, J. S. 2012b, MNRAS, 426, 2115

Konopacky, Q. M., Barman, T. S., Macintosh, B. A., \& Marois, C. 2013, Science, 339, 1398

Konopacky, Q. M., Macintosh, B., Marois, C., Barman, T., \& Ghez, A. 2010, Bulletin of the American Astronomical Society, 42, \#423.05

Konopacky, Q. M., Ghez, A. M., Barman, T. S., et al. 2009, Bulletin of the American Astronomical Society, 41, \#314.05

Krivov, A. V., Löhne, T., \& Sremčević, M. 2006, A\&A, 455, 509

Lagrange, A.-M., Boccaletti, A., Milli, J., et al. 2012, A\&A, 542, A40

Lafrenière, D., Marois, C., Doyon, R., \& Barman, T. 2009, ApJ, 694, L148

Löhne, T., Augereau, J.-C., Ertel, S., et al. 2012, A\&A, 537, A110

Lowrance, P.J., et al. 2005, AJ, 130, 1845

Marois, C., Zuckerman, B., Konopacky, Q. M., Macintosh, B., \& Barman, T. 2010, Nature, 468, 1080

Marois, C., Macintosh, B., Barman, T., et al. 2008, Science, 322, 1348

Marsh, K. A., Dowell, C. D., Velusamy, T., Grogan, K., \& Beichman, C. A. 2006, ApJ, 646, L77

Matthews, B. C., Sibthorpe, B., Kennedy, G., et al. 2010, A\&A, 518, L135

Melis, C., Zuckerman, B., Rhee, J. H., \& Song, I. 2010, ApJ, 717, L57

Moór, A., Ábrahám, P., Derekas, A., et al. 2006, ApJ, 644, 525

Moshir, M. \& et al. 1990, in IRAS Faint Source Catalogue, version 2.0 (1990), 0

Moro-Martín, A., Malhotra, R., Bryden, G., et al. 2010, ApJ, 717, 1123

Mouillet, D., Larwood, J. D., Papaloizou, J. C. B., \& Lagrange, A. M. 1997, MNRAS, 292, 896

Müller, S., Löhne, T., \& Krivov, A.V. 2010, ApJ, 708, 1728

Ott, S., 2010, in ASP Conference Series, Astronomical Data Analysis Software and Systems XIX, eds., Y. Mizumoto, K.-I. Morita, \& M. Ohishi

Patience, J., et al. 2011, A\&A, 531, 17

Perryman, M. A. C. \& ESA, eds. 1997, ESA Special Publication, Vol. 1200, The HIPPARCOS and TYCHO catalogues.

Astrometric and photometric star catalogues derived from the ESA HIPPARCOS Space Astrometry Mission

Pilbratt, G. L., Riedinger, J. R., Passvogel, T., et al. 2010, A\&A, 518, L1

Poglitsch, A., Waelkens, C., Geis, N., et al. 2010, A\&A, 518, L2

Quillen, A.C., \& Thorndike, S. 2002, ApJ, 578, 149

Raymond, S. N., Quinn, T., \& Lunine, J. I. 2004, Icarus, 168, 1

Reidemeister, M., Krivov, A. V., Schmidt, T. O. B., et al. 2009, A\&A, 503, 247

Rodriguez, D. R., \& Zuckerman, B. 2012, ApJ, 745, 147

Sadakane, K., \& Nishida, M. 1986, PASP, 98, 685

Sibthorpe, B., Vandenbussche, B., Greaves, J. S., et al. 2010, A\&A, 518, L130

Soummer, R., et al. 2011, ApJ, 741, 55.

Stapelfeldt, K. R., Holmes, E. K., Chen, C., et al. 2004, ApJS, 154,458 
Su, K. Y. L., Rieke, G. H., Stapelfeldt, K. R., Malhotra, R., Bryden, G., Smith, P. S., Misselt, K. A., Moro-Martin, A., \& Williams, J. P. 2009, ApJ, 705, 314

Su, K. Y. L., Rieke, G. H., Misselt, K. A., et al. 2005, ApJ, 628, 487

Sylvester, R. J., Skinner, C. J., Barlow, M. J., \& Mannings, V. 1996, MNRAS, 279, 915

Watson, C. A., Littlefair, S. P., Diamond, C., et al. 2011, MNRAS, 413, L71

Weinberger, A. J., Becklin, E. E., \& Zuckerman, B. 2003, ApJ, 584, L33

Williams, J. P. \& Andrews, S. M. 2006, ApJ, 653, 1480

Wilner, D. J., Holman, M. J., Kuchner, M. J., \& Ho, P. T. P. 2002, ApJ, 569, L115
Wright, D. J., Chené, A.-N., De Cat, P., et al. 2011, ApJ, 728, L20

Wyatt, M. C. et al. 2012, MNRAS, 424, 1206

Wyatt, M. C. 2008, ARA\&A, 46, 339

Wyatt, M. C. 2006, ApJ, 639, 1153

Wyatt, M. C. 2003, ApJ, 598, 1321

Wyatt, M. C., Dermott, S. F., Telesco, C. M., et al. 1999, ApJ, 527,918

Zuckerman, B., \& Song, I. 2004a, ApJ, 603, 738

Zuckerman, B., \& Song, I. 2004b, ARA\&A, 42, 685 